\title{
Case Management Berufsbildung - Eine Reform im Kontext der Subjektivierung von Erwerbsarbeit
}

\section{Katrin Kraus}

Eine wachsende Zahl von Jugendlichen hat Schwierigkeiten beim Einstieg in Ausbildung und Arbeitsmarkt, wodurch auch die gesellschaftliche Integrationsfunktion des Berufsbildungssystems geschwächt wird. Case Management Berufsbildung (CMBB) soll hier Abhilfe schaffen. Eine Argumentationsanalyse der entsprechenden Konzepte zeigt, dass CMBB in erster Linie mit sozialpolitischen Problemlagen sowie dem Hinweis auf Kapazitätsprobleme und Dysfunktionalitäten des Berufsbildungssystems begründet wird. Darüber hinaus ist die über CMBB vollzogene Subjektivierung der Berufsbildung im Kontext der Transformation der Erwerbsarbeit zu verstehen, da diese die Voraussetzungen gesellschaftlicher Integration grundlegend verändert. Notwendig ist daher auch ein erweitertes Verständnis der Übergangsproblematik.

Das Duale System der Berufsbildung galt lange als Garant für die gesellschaftliche Integration der jüngeren Generation. Mittlerweile haben Jugendliche aber auch in Ländern wie der Schweiz und Deutschland zunehmend Schwierigkeiten beim Einstieg in Ausbildung und Beschäftigung. Angesichts dieser Situation wird vielfach eine engere Verbindung zwischen Berufsbildung und Sozial- bzw. Sonderpädagogik gefordert und in Projekten erprobt (u.a. Biermann, 1996; Eckert, Heisler \& Nitschke, 2007; Friese \& Stöppler, 2009; Grimm \& Vock, 2007; Lex, Gaupp, Reißig \& Adamczyk, 2006; Lisop, 2009; Niemeyer, 2004). In grösserem Umfang wird diese Annäherung zurzeit in der Schweiz mit der Einführung von Case Management Berufsbildung (CMBB) in der Berufsbildung vollzogen.

Ausgehend von einer Bundesinitiative arbeiten 24 Kantone an der Konzeption bzw. Umsetzung von CMBB (Kraus, 2010). Die in diesem Rahmen vorgelegten kantonalen Konzepte ${ }^{1}$ bieten eine empirische Grundlage um aktuelle Veränderungen im Selbstverständnis der Berufsbildung anhand eines konkreten Materialkorpus nachzuvollziehen. Der vorliegende Beitrag basiert auf einer inhaltsanalytischen Untersuchung der CMBB-Konzepte unter der Fragestellung, wie die Einführung des CM-Ansatzes von Seiten der Berufsbildung 
thematisiert und begründet wird. Er stellt darüber hinausgehend Bezüge her zwischen der Einführung von einzelfallorientierten Ansätzen wie dem Case Management (CM) und veränderten Anforderungen an die Berufsbildung im Kontext einer Subjektivierung von Erwerbsarbeit, die die Bedingungen gesellschaftlicher Integration grundlegend verändert. Im Ausblick wird schliesslich ein erweitertes Verständnis von Übergängen vorgeschlagen.

\section{Integrationsfunktion, Schwellenproblematik und CM}

Die Entstehung der beiden pädagogischen Disziplinen Berufsbildung und Sozialpädagogik ist eng mit den politischen Veränderungen, sozialen Konflikten und ökonomischen Transformationen in Deutschland am Übergang vom 19. zum 20. Jhdt. verbunden. Vor diesem Hintergrund haben sich beide insbesondere die Integration (und Disziplinierung) der proletarischen Jugend in die neue, industriell-bürgerliche Gesellschaftsordnung zur Aufgabe gemacht (Böhnisch, 2005; Greinert, 1998; Schröer, 1999; Stratmann, 1992). Losgelöst von dieser konkreten Zielgruppe und dem spezifisch historisch-nationalen Kontext besteht für beide Teilbereiche der Pädagogik ein gesellschaftlicher Integrationsauftrag fort. Für beide ist die gesellschaftliche Erwartung konstitutiv, über entsprechende pädagogische Interventionen für Individuen resp. spezifische Zielgruppen gesellschaftliche Eingliederung und Teilhabe zu ermöglichen und damit den sozialen Zusammenhalt der Gesellschaft zu stärken. Im Selbstverständnis der Sozialpädagogik als «Pädagogik der sozialen Integration» (Böhnisch, 2005, S. 13) kommt dieser Anspruch explizit zum Ausdruck. Für die Berufsbildung ist ein Verständnis von sozialer Integration über berufliche Integration bis heute eine wichtige Grundlage, auch wenn ihre Realisierung zunehmend problematischer wird (Rützel, 2009).

In der traditionellen berufspädagogischen Terminologie handelt es sich bei den aktuellen Problemen um eine Verschärfung der Schwellenproblematik. Dieser Begriff beruht auf der Semantik des Hindernisses und markiert damit eine objektive und eine subjektive Seite der Problematik: Auf der einen Seite das zu überwindende Hindernis und auf der anderen Seite die individuellen Möglichkeiten und Ressourcen, die hierfür zur Verfügung stehen, sowie die Motivation, sich dieser Anstrengung zu unterziehen. Hindernisse stellen sich in Bezug auf die Berufsbildung insbesondere an den Übergängen zwischen den verschiedenen Bereichen und man unterscheidet traditionellerweise zwei Schwellen: Einstieg von der allgemeinen Bildung in die Berufsbildung und Übertritt in eine (angemessene) Erwerbstätigkeit (vgl. Grafik 1). 


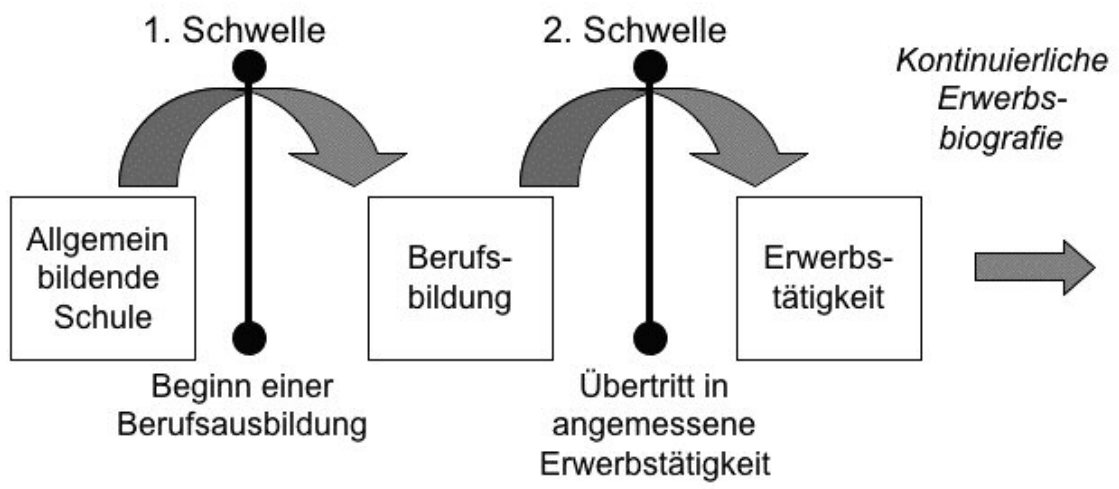

\section{Grafik 1: Die Schwellenproblematik in der Berufsbildung}

Die Berufsbildung stellt insgesamt eine Form der institutionellen Gestaltung des Übergangs von der Schule in die Erwerbsarbeit dar. Aufgrund der Verbindung von Berufsschule und Ausbildungsbetrieb in dualen Berufsbildungssystemen, d.h. der institutionellen Überlappung von Bildungs- und Beschäftigungssystem, sind die Schwellen hier relativ niedrig. Diese Systeme galten lange Zeit wegen der dadurch erreichten niedrigen Jugendarbeitslosigkeit als vorbildlich (z.B. Rauner, 2006). Allerdings ist auch hier das Überwinden der Schwellen im Zusammenhang mit der Berufsbildung problematischer geworden. Schaffner (2007) spricht daher bspw. von einer «risikoreichen Statuspassage» (S. 17). Einerseits bestehe ein hoher Verpflichtungsgrad, nach der obligatorischen Schulzeit eine «normale» Anschlusslösung zu finden, andererseits werde die individuelle Realisierung dieses Anspruchs aber immer schwieriger (ebd.).

Dies gilt in besonderem Masse für so genannte benachteiligte Jugendliche. Obwohl der Begriff der Benachteiligung sehr unterschiedlich verstanden wird (Bohlinger, 2004), sind vor allem Jugendliche mit so genanntem «Migrationshintergrund ${ }^{2}$ im Blick. Für sie ist nicht nur die erste Schwelle schwieriger zu überwinden als für Jugendliche der Schweizer Mehrheitsbevölkerung (Bertschy, Böni \& Meyer, 2007; Egger, Dreher \& Partner, 2007; Hupka \& Stalder, 2004; Meyer 2003), sondern auch der daran eigentlich anschliessende Übertritt in eine adäquate Beschäftigung. Der "Integrationsbericht» (Bundesamt für Migration $[B F M], 2006)$ hebt in diesem Zusammenhang hervor, dass die Erwerbslosenquote mit fast $9 \%$ bei Ausländern dreimal höher ist als bei Schweizern (ebd., S. 5). Im nachfolgenden «Bericht Integrationsmassnahmen» (BFM, 2007) stehen denn auch bei den insgesamt 45 aufgeführten Massnahmen zur Verbesserung der gesellschaftlichen Integration Bildung und Arbeit an erster Stelle. Die Berufsbildung wird dabei als einer der zentralen Bereiche hervorgehoben und direkt auf das gerade initiierte CMBB verwiesen, dessen «Integrationförderungspotenzial» (ebd., S. 12) es gezielt auszuloten gelte. 
Die Übergänge in Berufsbildung und Arbeitsmarkt und damit die soziale Integration über berufliche Bildung sind insgesamt prekär geworden, wodurch letztlich auch das Berufsbildungssystem als gesellschaftliche Institution geschwächt wird. Zudem resultieren aus der Problematik eines schwierigen Übergangs in die Berufsbildung individuelle, zielgruppenspezifische sowie gesellschaftliche Problemlagen. Es besteht also in mehrfacher Hinsicht Handlungsbedarf, der neben verschiedenen Massnahmen und Angeboten auf kantonaler Ebene auch zur Initiierung von $\mathrm{CMBB}$ durch das Bundesamt für Berufsbildung und Technologie (BBT) geführt hat.

Die Einführung von CMBB bedient sich eines aus der Einzelfallhilfe hervorgegangenen Ansatzes, der im pädagogischen Kontext bisher vor allem aus der Sozialpädagogik bekannt ist. Beim CM werden ausgehend von einem spezifischen Fall, d.h. einer konkreten Abklärung der spezifischen Problemlage einer Person, mögliche Ressourcen und notwendige Massnahmen koordiniert und ihre Umsetzung auf die vereinbarte Zielerreichung hin kontrolliert. Dies geht von einer/einem professionellen Case Manager/in aus und bedingt die Zusammenarbeit verschienender Institutionen, die konkret mit der im CM betreuten Person arbeiten. Im Rahmen der beruflichen Ausbildung wird in der Regel curricular-lernzielorientiert und nicht fallbezogen gearbeitet, daher stellt CMBB einen neuen Ansatz dar und kann auch als Annäherung der Berufsbildung an die Sozialpädagogik verstanden werden. Um diesen Annäherungsprozess vor dem Hintergrund veränderter Anforderungen an die Berufsbildung im Kontext einer Transformation der Erwerbsarbeit diskutieren zu können, wird im Folgenden zunächst der Frage nachgegangen, wie die Einführung von CM von Seiten der Berufsbildung selbst begründet wird.

\section{Warum soll CM in der Berufsbildung eingeführt werden? Analyseergebnisse 3}

\section{Zur Grundierung der Argumentation}

Ausgangspunkt der Argumentation ist eine Vergewisserung über die Problematik des Übergangs in Berufsbildung und Arbeitsmarkt. Der Bund hat im Vorfeld der Entscheidung zur Initiierung von $\mathrm{CMBB}$ eine Studie zur Problematik des Übergangs in die Berufsbildung (Egger, Dreher \& Partner, 2007) in Auftrag gegeben, die hinter der eidgenössischen Initiative zur Lancierung von CMBB steht und in mehreren kantonalen Konzepten aufgegriffen wird. Die Autoren konstatieren, dass ca. 2,5-3\% der Schulabgänger/innen trotz bestehender Unterstützungsangebote erhebliche Schwierigkeiten am Übergang zur Berufsbildung haben und schlussfolgern daraus einen strategischen Handlungsbedarf (ebd., S. 6f.). Sie fordern u.a. eine frühzeitige Identifikation von Personen «mit zu erwartenden Problemen» (ebd., S. 7), eine systematische Erfassung von «allen Personen ohne Anschlusslösung» (ebd.) sowie die Etablierung von Unterstüt- 
zungsmodellen mit einer «langfristig ausgerichteten Fallführung vor, am und nach dem Übergang» (ebd.).

Neben den beiden allgemeinen Zielen des CMBB «Hilfe zur Selbsthilfe» und «Effizienzsteigerung» wird vom BBT (2007) für den Einzelfall das Erfolgskriterium "post-obligatorischer Abschluss" gesetzt (ebd., S. 1). Dies steht in Zusammenhang mit dem bildungspolitischen Beschluss «bis ins Jahr 2015 unter den 25-Jährigen den Anteil der Absolventinnen und Absolventen mit einem Abschluss auf der Sekundarstufe II auf 95\% zu steigern» (BBT \& Schweizerische Berufsbildungsämter-Konferenz [SBBK], 2008, S. 2). Diese outputorientierte Zielvorgabe für die künftige Entwicklung des Bildungssystems ist im Kontext internationaler Entwicklungen zu sehen, wo Akteure wie EU oder OECD solche Kennziffern als Benchmarks für den Vergleich von nationalen Bildungssystemen nutzen. Im Unterschied zu anderen Ländern stellt die Berufsbildung in der Schweiz traditionell einen wichtigen Weg dar, auf dem ein post-obligatorischer Abschluss auf Sekundarstufe II erworben wird.

Die Ergebnisse und Forderungen der erwähnten Studie von Egger u.a. bilden zusammen mit den bildungspolitischen Zielvorgaben die Hintergrundfolie, auf der das CMBB angestossen und konzeptionalisiert wird: Die Zahl der Jugendlichen ohne (berufliche) Ausbildung wird in beiden Zugängen als Problem thematisiert zu dessen Lösung CM eingeführt werden soll. Über diese argumentative Grundierung hinausgehend finden sich in den Konzepten explizite Begründungen für die Einführung von CMBB, die unterschiedliche Referenzpunkte ansprechen und sich zu den vier Argumentationsmustern legalistische, selbstreferentielle, gesellschaftsbezogene und individuumsbezogene Argumentation bündeln lassen. Die meisten Konzepte verweisen zudem auf bereits bestehende kantonale Aktivitäten im Problemfeld des Übergangs von der obligatorischen Schule in weitere Bildungsangebote bzw. den Arbeitsmarkt und thematisieren das Aufgreifen der Bundesinitiative als sinnvolle Fortführung ihres diesbezüglichen Engagements.

Das “argumentative Gespann» aus Legalistischer und selbstreferentieller Argumentation

Die legalistische Argumentation wird vom Bund bei der Initiierung von CMBB verfolgt. Im BBT-Konzept wird einleitend darauf hingewiesen, dass die Initiative sich auf die Artikel 3a, 3c, 7 und 12 des Berufsbildungsgesetzes (BBG) stützt und auf dieser Grundlage einen Rahmen für die Förderung von CMBB der Kantone festlegt (BBT, 2007, S. 1). Vor allem Artikel 3 formuliert den gesetzlich verankerten Integrationsauftrag der Berufsbildung: Ermöglichung von beruflicher und persönlicher Entfaltung, Integration in Arbeitsmarkt und Gesellschaft sowie Ausgleich von Bildungschancen. Dieser Auftrag wird im BBTKonzept durch den Verweis auf die entsprechenden Passagen im BBG rekapituliert und damit eine entsprechende Legitimation für die Initiative zur Lancierung von CMBB gegeben. 
Die kantonalen Dokumente beziehen sich wiederum auf unterschiedliche Referenzpunkte um die Einführung des $\mathrm{CMBB}$ zu begründen. In einem engen Zusammenhang mit der legalistischen Argumentation des Bundes steht die selbstreferentielle Argumentation in den kantonalen Konzepten. Im diesem Rahmen werden Probleme in verschiedenen Bereichen des Berufsbildungssystems ausgeführt und eine Besserung durch CMBB in Aussicht gestellt. Die Referenzpunkte der Argumentation liegen hier also innerhalb des Berufsbildungssystems selbst. Die legalistische und die selbstreferentielle Argumentation sind in ihrer Aussagekraft aufeinander angewiesen und bilden sozusagen ein «argumentatives Gespann». Es gibt einen gesetzlich verankerten Integrationsauftrag der Berufsbildung, der mit den jetzigen Strukturen nicht erfüllt werden kann. Mit diesem argumentativen Gespann werden diskursiv strukturelle Veränderungen des Systems abgesichert.

Im Mittelpunkt der Problematisierung des bestehenden Systems in den kantonalen Konzepten steht das Fehlen einer langfristigen, übergreifenden Strategie zur Eingliederung der Jugendlichen in die Berufsbildung bzw. das Bildungs- oder Beschäftigungssystem (AG, S. 26; BE, S. 5; BL, S. 6; JU, S.7; NE, S. 23; SG, S.8; SO, S.7; TG, S.6; Zentralschweizer BerufsbildungsämterKonferenz [ZBK], S.6; ZH, S.7). Stattdessen gebe es eine unübersichtliche und der Eingliederung wenig dienliche Vielfalt an Angeboten, die neben- und nacheinander stattfinden. Dies führe auch zu «Doppelspurigkeiten» auf der Seite der Angebote (BE, S. 28; NE, S. 2; SG, S. 7; TG, S. 17; ZBK, S.6), die in Einzelfällen sogar von Personen zum «Tourismus» zwischen den Angeboten genutzt würden (NE, S. 17; ähnlich SG, S. 7; TG, S. 16). Zudem finde teilweise eine zweckentfremdete Nutzung statt, z.B. wenn «Mittelschulen von schulstärkeren Jugendlichen ... als 'Zwischenlösung' missbraucht werden» (SH, S. 8). Gleichzeitig seien aber auch Lücken in der Versorgungsstruktur (z.B. AG, S. 25; ZBK, S. 18) zu erkennen, die verhindern, dass Personen mit adäquaten Massnahmen versorgt werden. Darüber hinaus wird bemängelt, dass Personen entweder aus eigenem Antrieb «abtauchen» (BL, S. 4; SH, S.15; ZH, S.7) oder aufgrund fehlender Zuständigkeiten aus dem System «herausfallen « (BL, S. 18; SG, S. 7; TG, S. 18; ZH, S. 5; ähnlich SO, S. 6). Daraus resultiert das Problem, dass sich die Jugendlichen nicht in einer Institution des Bildungssystems befinden.

Ein zweites zentrales Thema ist der selbstreferentiellen Argumentation die fehlende Koordination bestehender Angebote, eine unklare Verteilung von Verantwortlichkeiten zwischen den verschiedenen Institutionen sowie eine mangelnde Zusammenarbeit der zuständigen politischen Akteure (BE, S. 5; BS, S. 6; JU, S. 7; NE, S. 2; SG, S. 7; SH, S. 5; SO, S. 7; TG, S. 4; ZH, S. 8). Die Kantone streben zur Abhilfe die Initiierung bzw. Verbesserung der «interinstitutionellen Zusammenarbeit» an und beziehen sich auf die Zusammenarbeit zwischen: der allgemein bildenden Schule und der Berufsbildung; der Berufsbildung und den Institutionen der Arbeitslosen-, Invaliditäts- und Sozialversicherung sowie das Zusammenwirken von Berufsberatung und -bildung. In 
Bezug auf die Angebote in der Berufsbildung möchte man zudem Synergien nutzen (BE, S. 28; GE, S. 1; SO, S. 9; TG, S. 4) und die Jugendlichen «nicht nur ressourcensparender, sondern wirksamer» (ZBK, S. 13; ähnlich NE, S. 2) unterstützen. Es geht also insgesamt um strukturelle Veränderungen in der Berufsbildung gemäss den Leitlinien Effektivität und Effizienz.

Ein dritter Bereich der selbstreferentiellen Argumente befasst sich schliesslich mit Kapazitätsproblemen des jetzigen Systems. Neben der Tatsache, dass zu wenige Lehrstellen, insbesondere mit niedrigeren Anforderungen, bestehen (AR, S. 7; GR, S. 3; JU, S.7; NE, S. 25; SH, S. 6; ZBK, S. 3) sowie vereinzelten Hinweisen auf eine eingeschränkte Lehrstellenvielfalt (GR, S. 4) bzw. das Passungsverhältnis von Angebote und Nachfrage nach Lehrstellen (NE, S. 25; SO, Anhang), werden Überlastungsphänomene thematisiert. Eine Be- bzw. Überlastung von Lehrpersonen und Schulen (AR, S. 7; SH, S. 8; ZBK, S. 19), Lehrbetrieben (GR, S. 4; SO, S. 8; ZBK, S. 19) sowie der Berufsinspektorate (GR, S. 5) bzw. Berufsberatung (ZBK, S. 19) entstehe durch einen generell gestiegenen Beratungsbedarf der Jugendlichen (SO, Anhang; VD, S. 1) sowie insbesondere durch Jugendliche mit Problemen.

Die Einführung von CMBB soll also Mängel in den Bereichen Strategie, Koordination und Kapazitäten beheben und somit über eine Strukturveränderung die Funktionalität der Berufsbildung in Bezug auf ihre im BBG festgehaltene Integrationsfunktion wiederherstellen. Die klare Verteilung der beiden Argumente im argumentativen Gespann von legalisitischer und selbstreferentieller Argumenation auf die eidgenössische und kantonale Ebene spiegelt die Kompetenzverteilung zwischen beiden Akteuren: In der Berufsbildung obliegt dem Bund die Rahmengesetzgebung sowie die strategische Planung und Entwicklung während die Kantone für die Umsetzung, d.h. die Gestaltung einer kantonale Gesetzgebung und Angebotsstruktur, verantwortlich sind.

\section{Das Spektrum zwischen gesellschafts- und indivuumsbezo- genen Argumenten}

Die gesellschaftsbezogene und die individuumsbezogene Argumentation innerhalb der CMBB-Konzepte sind ebenfalls miteinander verknüpft, da beide im Verhältnis von Individuum und Gesellschaft angesiedelt sind. Sie finden sich in unterschiedlicher Ausprägung in den kantonalen Konzepten.

Die gesellschaftsbezogene Argumentation tritt in einer materiellen und einer immateriellen Variante auf. Bei der ersten steht die Einsparung öffentlicher Mittel im Vordergrund, wobei der Bezugspunkt primär die Kosten der sozialen Sicherung sind (AG, S. 4; BE, S. 6; BL, S. 3; BS, S. 4; GR, S. 5; SO, S. 10 u. Anhang; TG, S. 22; ZBK, S. 19; ZH, S. 5). Im Konzept der Zentralschweizer Kantone werden darüber hinaus die unspezifischen Kosten erwähnt, die einer Gesellschaft durch «soziale Verwahrlosung» (ZBK, S. 21) entstehen. Das Grundargument lautet: Jugendliche mit Übergansproblemen «verursachen in der Folge als Erwachsene mit hoher Wahrscheinlichkeit weitere Kosten (Arbeitslosigkeit, 
Sozialhilfe, Invalidität etc.)» (AG, S. 5) und stellen somit «für die Gesellschaft ein hohes finanzielles Risiko dar» (BL, S. 7). Die in diesem Bereich entstehenden Kosten könnten - so die weitere Argumentation - durch ein frühzeitiges und systematisches Eingreifen auf der Basis von $\mathrm{CMBB}$ verringert oder sogar vermieden werden.

Die Bezugspunkte der - weniger stark akzentuierten - immateriellen Argumentation sind gesellschaftliche Integration und sozialer Zusammenhalt, wobei hier gemäss der berufspädagogischen Perspektive der Arbeitsmarkt als Zugang zur Gesellschaft eine hervorgehobene Rolle einnimmt. Berufsbildung biete «die besten Chancen zur Integration in die Gesellschaft» (SO, Anhang; ähnlich ZH, S. 8) und zur Verhinderung von Marginalisierung (VS, S. 13). $\mathrm{CMBB}$ wird als Möglichkeit der «Erhöhung der Beteiligung junger Menschen an der Berufsbildung» (BS, S. 23) gesehen und damit als Antwort auf die "Herausforderungen einer komplexen und arbeitsteiligen Gesellschaft»(BE, S. 28) sowie die "grosse soziale Herausforderung" (SO, Anhang) durch eine "desintegration socioprofessionelle» (VD, S. 5). Lange Erwerbslosigkeit im jugendlichen Alter habe zudem nachteilige Effekte auf dem Arbeitsmarkt (BL, S. 10; TG, S. 3; ZH, S. 4) und die Steigerung der Abschlussquote liege deshalb im Interesse von Wirtschaft und Gesellschaft (ZBK, S. 4).

Die individuumsbezogene Argumentation thematisiert auf der einen Seite eine «Mitwirkungspflicht» (BS, S. 14; TG, S. 4) und auf der anderen Seite «einen Anspruch auf einen Abschluss der Sekundarstufe II» (BL, S. 3). Zusätzlich wird die Ermöglichung eines selbstverantwortlich geführten Lebens (BE, S. 28) als Chance für Jugendliche gesehen und fehlende Berufsausbildung als Armutsrisiko hervorgehoben (ZBK, S. 3). Zu den genannten Aspekten kommt noch eine entwicklungspsychologisch fundierte Argumentation, die die negativen Auswirkungen von Erwerbslosigkeit und Desintegration im Jugendalter für die weitere Entwicklung zur Sprache bringt (BL, S. 12; NE, S. 13; SO, Anhang). Dieser Sicht entspricht auch der Hinweis, dass man «den richtigen Zeitpunkt zum Eingreifen» (TG, S. 18; ähnlich ZBK, S. 8) nicht verpassen dürfe.

Bei diesem Argumentationszusammenhang wird also primär das für die Pädagogik zentrale Verhältnis von Individuum und Gesellschaft angesprochen. In Bezug auf das Individuum werden seine Entwicklung sowie vor allem seine Rechte und Pflichten in Bezug auf die Gesellschaft thematisiert, die durch CMBB besser realisiert werden könnten; bei der Gesellschaft geht es um ihre materielle und immaterielle Gefährdung durch (desintegrierte) Individuen und die Möglichkeit, dies durch CMBB zu verhindern. Die Argumente rekurrieren somit auf die für die Pädagogik konstitutive Funktion der Vermittlung im Verhältnis von Individuum und Gesellschaft, wobei der Schwerpunkt auf der berufspädagogischen Version liegt, d.h. der Erwerbstätigkeit als Zugang zur Gesellschaft. 


\section{Fazit zur Argumentationsanalyse}

Mit diesen vier Argumentationsmustern wird vor dem Hintergrund der Problemsicht auf Jugendliche ohne post-obligatorischen Abschluss die Notwendigkeit einer Einführung von CMBB begründet. Wie bereits durch den Umfang der Ausführungen deutlich wird, dominiert die selbstreferentielle Argumentation. Mehrheitlich wird als Ausgangspunkt für die Einführung vom CMBB dargelegt, dass das System der Berufsbildung in der jetzigen Form den gesellschaftlichen Erwartungen nicht (mehr) gerecht werden kann. Der für sie konstitutiven Erwartung, Jugendliche zu einem post-obligatorischen, beruflichen Abschluss zu führen und ihnen damit zur beruflichen und sozialen Integration zu verhelfen, kann die Berufsbildung mit den bisherigen Strukturen nicht gerecht werden - so das zentrale Argument - und braucht daher strukturelle Innovationen. Die Analyse der Argumentation zur Einführung des einzelfallbezogenen CMBB zeigt, dass es vor allem um die Wiederherstellung der Funktionalität des Berufsbildungssystems innerhalb eines bestehenden gesellschaftlichen Integrationsauftrags geht sowie um eine Vergewisserung der pädagogischen Aufgabe in der Vermittlung zwischen Individuum und Gesellschaft, insbesondere in Bezug auf den gesellschaftlichen Teilbereich Erwerbsarbeit. Dass diese Aspekte aktuell überhaupt zum Problem geworden sind, lässt sich auch auf eine Veränderung der grundlegenden Bedingungen für gesellschaftliche Integration über Berufsbildung resp. die Vermittlung von Individuum und Gesellschaft im Medium beruflicher Bildung zurückführen.

\section{CMBB als Reform der Berufsbildung im Kontext der Transformation der Erwerbsarbeit}

Wendt (2007, S. 460) konstatiert, dass CM allgemein im Rahmen von Restrukturierungen im Sozial- und Bildungsbereich an Bedeutung gewinnt, was man auch an der Einführung von CMBB nachvollziehen kann. ${ }^{4}$ Mit dem CM-Ansatz geht generell die Aussicht auf mehr Effektivität und Effizienz einher (z.B. Kleve, 2006), die man auch für die Berufsbildung anstrebt.

Darüber hinaus hofft man von Seiten der Bildungspolitik die Integrationsfunktion der Berufsbildung wieder besser $\mathrm{zu}$ realisieren und so auch zur (Wieder-)Absicherung des Berufsbildungssystems als gesellschaftlicher Institution beizutragen. Beim CMBB geht es nicht nur darum, das «Integrationsförderungspotenzial» für Jugendliche mit Migrationshintergrund auszuloten, sondern genereller um die Frage des sozialen Zusammenhalts, d.h. um das, "was eine Gesellschaft zusammenhält», und darum, welches Mass an Desintegration sie verträgt. Neben den Instrumenten der sozialen Sicherung ist damit auch die Bildung angesprochen - mit Becker und Lauterbach (2007) also die «soziale Frage des 21. Jahrhunderts» (S. 429). 
Bezogen auf das Bildungssystem intendiert CMBB eine (Re)Strukturierung des Übergangsbereichs von der allgemeinen in die berufliche Bildung. Dieses Segment hat sich bisher weitgehend massnahmenförmig entwickelt und ist noch nicht als eigener Bereich des Bildungssystems institutionalisiert. Die Einführung von $\mathrm{CMBB}$ zielt auf eine Regulierung dieses Bereichs im Schnittfeld von Bildung, Arbeitsmarkt und Sozialer Sicherung. Das Anliegen der Wiederherstellung ihrer Funktionalität durch (Re)Organisation von pädagogischen Unterstützungsprozessen wird somit begleitet von einer tendenziellen Ausweitung der institutionellen Reichweite der Berufsbildung.

Die aktuellen Schwierigkeiten in Bezug auf die berufliche Integration verweisen auf beide Seiten der eingangs erwähnten Schwellenproblematik. Wenn an den Schwellen zum Einstieg in Berufsbildung und Beschäftigung die Hürden höher werden, muss zu ihrer Überwindung auf der subjektiven Seite ein Mehr an Motivation, Ressourcen und Fähigkeiten vorhanden sein. Die Erhöhung der Hürden ist teilweise auf gestiegene fachliche Anforderungen zurückzuführen, liegt aber auch in veränderten Erwartungen an die Selbstregulierungs- und -gestaltungsfähigkeit innerhalb flexibilisierter, entgrenzter und subjektivierter Erwerbsarbeit (z.B. Kratzer, 2003; Moldaschl \& Voss, 2003).

Im Kontext der Subjektivierung von Erwerbsarbeit wird die Bereitschaft zur permanenten "Arbeit an sich selbst» zur Grundlage einer Befähigung zur Erwerbsarbeit (Kraus, 2006, S. 71). Diesem Anspruch wird von der Mehrzahl der Individuen selbstreguliert entsprochen, d.h. sie sind in der Lage, ihre Selbstkompetenzen selbstreguliert und ihm Rahmen bestehender Bildungsangebote zu steigern. Zum Teil macht der höhere Anspruch an Selbstregulierung aber einen spezifischen Support erforderlich. Dieser geht über die traditionelle Arbeitsweise der Berufsbildung hinaus, da er neben individualisierten Unterrichtsformen auch stärker subjektbezogene und einzelfallorientierte Massnahmen erfordert, die bisher vor allem in pädagogischen Feldern ausserhalb des Bildungssystems verankert sind. Die Berufsbildung eignet sich hier eine eher sozialpädagogische Arbeitsweise an und reagiert so mit einer Subjektivierung ihrer pädagogischen Praxis auf die veränderten Anforderungen an erwerbsorientierte Pädagogik im Übergang zu post-industriellen Gesellschaft, die sich neben den fachlichen Anforderungen insbesondere auf die Selbstkompetenzen ihrer Absolvent/innen beziehen.

\section{Ausblick - ein erweitertes Verständnis der übergangsproblematik}

Über die Suche nach problemlösenden Ansätzen beim Einstieg in die Berufsbildung hinaus ist angesichts veränderter gesellschaftlicher Rahmenbedingungen ein Überdenken grundlegender Modelle notwendig. Zur Disposition steht dabei insbesondere das Modell des Übergangs als Überwindung von Schwellen beim 
Eintritt in neue Phasen des Lebenslaufs. Denn «der 'Gegenstand', auf den sie [die Übergangsforschung] sich bezieht, wird zunehmend komplexer und fluider» (Kutscha, 2008, S. 71). Demzufolge ist die Übergangsproblematik nicht mehr auf die Einmündung in Berufsbildung und Erwerbstätigkeit fokussiert. Eine erfolgreiche Bewältigung von Übergängen zwischen Bildung und Erwerbstätigkeit stellt sich nicht (mehr) als punktuelle Herausforderung in einem institutionell vorstrukturierten Lebenslauf, die bei Bedarf mit spezifischen Unterstützungsmassnahmen flankiert werden kann. Diese Vorstellung wird vielmehr abgelöst von derjenigen biografisch dislozierter Übergänge, die im Laufe des (Erwerbs)lebens von den Einzelnen zu bewältigen sind. Dies führt zu einem erweiterten Modell der Schwellenproblematik (vgl. Grafik 2), das die Bewältigung von Übergängen zwischen und innerhalb von (Berufs)Bildung und Erwerbstätigkeit als lebenslaufbegleitende Anforderung an die Individuen versteht und sie damit über die Benachteiligtenförderung hinaus zum Gegenstand beruflichen Lernens macht.

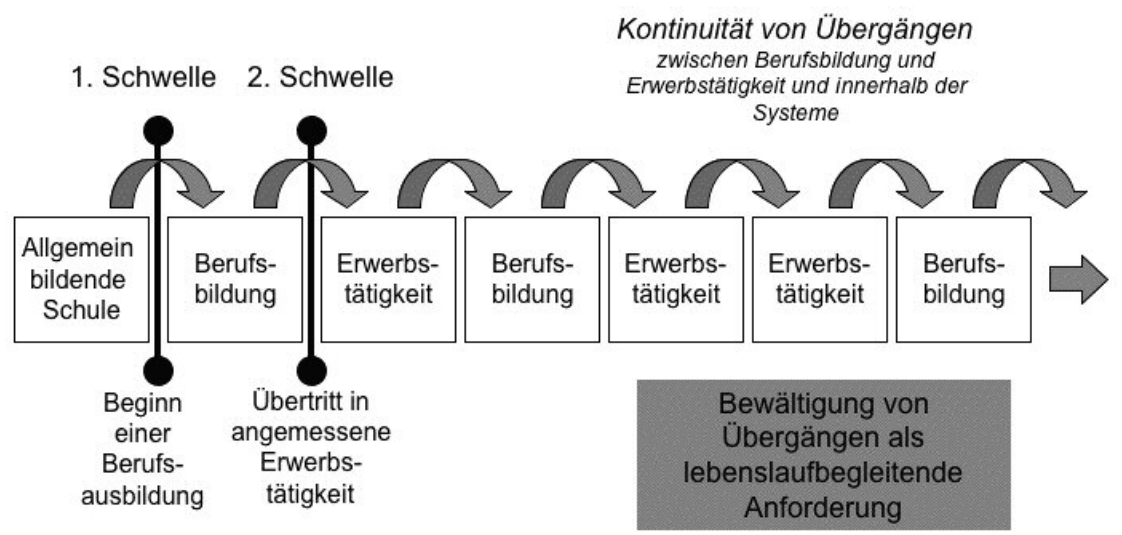

Grafik 2: Ein erweitertes Modell der Schwellenproblematik in der Berufsbildung

Unter dieser Perspektive stellt sich in Bezug auf die Schwellenproblematik eine doppelte Herausforderung: Das Überwinden der beiden traditionellen Schwellen wird offensichtlich problematischer, daher versuchen institutionelle und pädagogische Arrangements hier mit verschiedenen Interventionen spezifische Hilfestellungen zu geben. ${ }^{5}$ Gleichzeitig lässt sich die Überwindung der Schwellen angesichts der Kontinuität der Übergänge nicht mehr nur am Beginn des Erwerbslebens lokalisieren, sondern wird zu einer permanenten Aufgabe angesichts eines flexibilisierten Arbeitsmarkts, einer Politik des Lebenslangen Lernens und einer Destandardisierung von Lebensläufen. Die individuelle Kompetenz, Übergänge zwischen Bildungs- und Beschäftigungssystem zu meistern und die eigene Erwerbsbiografie aktiv zu gestalten wird damit zu einem neuen Aspekt erfolgreicher Erwerbstätigkeit bzw. zur neuen Aufgabe im Erwach- 
senenalter und gewinnt daher auch für die berufliche Bildung eine neue Relevanz (Hendrich, 2005; Kraus, 2008). Für die Berufsbildung ist es daher wichtig eine Perspektive zu entwickeln, die der Stärkung der Handlungsfähigkeit des Einzelnen in einer zunehmend flexiblen Erwerbswelt verpflichtet ist. Dabei muss sie einerseits im Rahmen berufspädagogischer Regelangebote die Kontinuität der Gestaltung von Übergängen aufgreifen und andererseits gleichzeitig über Spezialisierung und Differenzierung der Tatsache Rechnung tragen, dass die dabei zu überwindenden Hürden unter den Bedingungen gesellschaftlicher Ungleichheit unterschiedlich hoch sind und die Individuen nicht über die gleichen (materiellen wie immateriellen) Ressourcen ihrer Überwindung verfügen.

\section{Anmerkungen}

1 Die Dokumente sind zugänglich über: http://edudoc.ch/collection/casemanagement?ln=de (Zugriff am 20.03.2010)

2 Es gibt keine generelle Operationalisierung des Kriteriums "Migrationshintergrund", dennoch hat sich der Begriff - mangels besserer Alternativen - in den Sozialwissenschaften durchgesetzt und wird auch in der Berufsbildung verwendet (z.B. Bundesinstitut für Berufsbildung, 2009, S. 163). Neben der Staatsangehörigkeit - die aufgrund ihrer guten Operationalisierbarkeit in Statistiken häufig zugrunde gelegt, aber der Komplexität der Thematik nicht gerecht wird - erfasst er Variablen wie Geburtsland der Eltern oder die Sprache, die in der Familie primär gesprochen wird.

3 Die folgenden Ausführungen basieren auf einer inhaltsanalytischen Untersuchung des BBT-Konzepts sowie der kantonalen Konzepte zum CMBB. Die 24 kantonalen Konzepte bestehen z.T. aus mehreren Dokumenten. Die Hauptdokumente (sowie ggf. zusätzlich Anhänge/Beigaben) wurden in Bezug auf die Argumentation einzeln und vollständig mit einer dreistufigen Inhaltsanalyse (Identifizieren, Dokumentieren und Paraphrasieren) untersucht, wobei die Kategorien induktiv gebildet wurden. Das italienischsprachige Konzept (Tessin) konnte aus sprachlichen Gründen nicht einbezogen werden. Eine grobe Durchsicht zeigt aber keine grundlegend neuen Aspekte.

4 Die Verbreitung von Konzepten wie CM lässt sich aber nicht nur funktional begründen. Sie weist vielmehr Ähnlichkeiten zu dem von Link (Link, 1986; Link \& Link-Heer, 1990) entwickelten Ansatz des «Interdiskurses» auf, d.h. eines Diskurses, der zwischen verschiedenen Spezialdiskursen vermittelt und sie koppelt bzw integriert. Interdiskursen liegen Kollektivsymbole und Dispositive zugrunde. Im Falle des CM ist das die «Vertraglichkeit», d.h. die Vorstellung, dass soziale Beziehungen auf einer wechselseitigen Verpflichtung und im weitesten Sinne einer gegenseitigen Leistungserbringung basieren, z.B. indem das Individuum über eine vertragsähnliche Beziehung in eine Problemlösung eingebunden wird.

5 Hierbei zeigt sich allerdings ein Problem pädagogischer Intervention im Kontext sozialer Probleme: auf der einen Seite braucht es gerade angesichts widriger Umstände eine optimale und auf den Einzelfall zugeschnittene Unterstützung um bessere Lern- und Entwicklungsverläufe und damit auch soziale Chancen zu ermöglichen, auf der anderen Seite lassen sich aber damit (allein) gesellschaftlich induzierte Probleme nicht lösen. 


\section{Literatur}

\section{Quellen}

AG (=Departement Bildung, Kultur und Sport, Abteilung Berufsbildung und Mittelschule des Kantons Aargau): Case Management Berufsbildung im Kanton Aargau: Am Übergang von der obligatorischen Schule in die Berufsbildung. Grundlagenpapier. 04.10 .07

AR (=Department Bildung, Amt für Mittel- und Hochschulen und Berufsbildung des Kantons Appenzell Ausserrhoden): Case Management AR. Fachkundige individuelle Begleitung AR. Konzept. August 2007

BBT (=Bundesamt für Berufsbildung und Technologie) (2007): Case Management Berufsbildung. Grundsätze und Umsetzung in den Kantonen. Bern. 22.02.07

BE (=Kanton Bern): Take off ... erfolgreich ins Berufsleben! Case Management BerufsBildung CM BB. Kantonales Gesamtkonzept (Schlussbericht zum Vorprojekt). 28.08.07

BL (=Bildungs-, Kultur- und Sportdirektion; Finanz- und Kirchendirektion; Volkswirtschafts- und Sanitätsdirektion Kanton Basel-Landschaft; Arbeitsgruppe Interinstitutionelle Zusammenarbeit): Strategien gegen die Erwerbslosigkeit Jugendlicher im Kanton Basel-Landschaft. Bericht der Arbeitsgruppe Interinstitutionelle Zusammenarbeit. 08.02.07

BS (=Wirtschafts- und Sozialdepartement und Erziehungsdepartement des Kantons BaselStadt/Rolf Schürmann und Benedikt Arnold, Projektleitung Strategiegruppe Jugendarbeitslosigkeit, Leitung Projektgruppe GAP unter Mitwirkung von Christoph Marbach, Amt für Berufsbildung und Berufsberatung): Gesamtkonzept Case Management Berufsbildung. Projekt GAP, Case-Management Berufsbildung. 01. 07.07

FR (=Direction de l'économie et de l'emploi/Volkswirtschaftsdirektion - Service de la formation professionelle/Amt für Berufsbildung; Groupe de travail interdirectionnel des Kantons Fribourg/Service de la formation professionelle): Case Mangement - Canton Fribourg - Concept global. 07.09.07/10.12.07

GE (=Office pour l'orientation, la formation professionelle et continue): Gestion du suivi individualisé «formation professionelle». Case management «formation professionelle». Concept genevois. o.D.

GR (=Kanton Graubünden): Case Management Berufsbildung. Integration von Jugendlichen mit schulischen, sozialen und sprachlichen Schwierigkeiten in der Berufsbildung. 25.05 .07

JU (=Departement de la formation, de la culture et des sports; service de la formation des niveaus secondaire II et tertiaire): La case mangement. Concept global pour le canton du Jura. o.D.

NE (=Kanton Neuchâtel): Le case management dans la formation professionelle. Concept du canton de Neuchâtel. August 2007/27.11.07

SG (=Amt für Berufsbildung des Kantons St. Gallen): Case Management Berufsbildung. Gesamtkonzept Kanton St.Gallen. 07.09.07

SH (=Berufsbildungsamt des Kantons Schaffhausen): Case Management Berufsbildung. Rahmenkonzept Kanton Schaffhausen. 31.08.07

SO (=Amt für Berufsbildung und Berufsberatung des Kantons Solothurn): Kantonales Gesamtkonzept August 2007. Case Management Berufsbildung. Der Kanton Solothurn koordiniert gegen Jugendarbeitslosigkeit. 28.08.07

TG (=Kanton Thurgau/Anders Stokholm): Rahmenkonzept Thurgau Case Management Berufsbildung. Bericht über die Koordination der vorhandenen Angebote zur Begleitung von durch Arbeitslosigkeit bedrohten Jugendlichen. o.D. (Sommer 2007)

TI (=Kanton Tessin): «Case Mangement nella Formazione Professionale» Ticino - Progetto 2007/2008 (con possibile estensionle fino al 2011). Integratzione nelle formazione 
proessionale di giovani a rischio con difficoltà scolastiche, sociale e linguistiche. 31.08 .07

VD (=Kanton Vaud/Waadt): Concept cantonal vaudois de case management «formation professionelle». o.D.

VS (=Kanton Valais/Wallis/Nasca Formation): Concept de Case Management «formation profesionelle» - Canton du Valais. 16.08.07

ZBK (=Zentralschweizer Berufsbildungsämter-Konferenz): Konzept «Case Management Berufsbildung in der Zentralschweiz». Vorprojekt im Rahmen der BBT-Initiative. 22.06.07

ZH (=Bildungsdirektion Kanton Zürich, Amt für Jugend und Berufsbildung / Kurt Häfeli, Leiter Forschung \& Entwicklung Interkantonale Hochschule für Heilpädagogik (HfH) Zürich): Case Management Berufsbildung (CM BB). Gesamtkonzept für den Kanton Zürich. 07.09.07

\section{Sekundärliteratur}

Becker, R. \& Lauterbach, W. (2007). Bildung als Privileg. Erklärungen und Befunde zu den Ursachen der Bildungsungleichheit. Wiesbaden: VS Verlag.

Bertschy, K., Böni, E. \& Meyer, T. (2007). An der zweiten Schwelle: Jugendliche beim Übergang in Ausbildung und Arbeitsmarkt. Ergebnisse des Jugendlängsschnitts TREE, Update 2007. Bern: TREE.

Biermann, H. (1996). Benachteiligte im Spannungsfeld von Berufsbildung, Sozialpädagogik und Rehabilitation. Zeitschrift für Berufs- und Wirtschaftspädagogik, 92, 81-85.

Böhnisch, L. (2005). Die Sozialpädagogik der Lebensalter. Weinheim/München: Juventa.

Bohlinger, S. (2004). Der Benachteiligtenbegriff in der Berufsbildung. Zeitschrift für Berufsund Wirtschaftspädagogik, 100, 230-241.

Bundesamt für Berufsbildung und Technologie \& Schweizerische BerufsbildungsämterKonferenz (2008). Case Management Berufsbildung. Unterstützung und Begleitung der Umsetzung in den Kantonen. Projektauftrag BBT-SBBK.

Bundesamt für Migration (2006). Probleme der Integration von Ausländerinnen und Ausländern in der Schweiz. Bestandsaufnahme der Fakten, Ursachen und Risikogruppen, Massnahmen und des integrationspolitischen Handlungsbedarfs. Bern.

Bundesamt für Migration (2007). Bericht Integrationsmassnahmen. Bericht über den Handlungsbedarf und die Massnahmenvorschläge der zuständigen Bundesstellen im Bereich der Integration von Ausländerinnen und Ausländern per 30. Juni 2007. BernWabern.

Bundesinstitut für Berufsbildung (2009). Datenreport zum Berufsbildungsbericht 2009. Bonn.

Eckert, M., Heisler, D. \& Nitschke, K. (2007). Sozialpädagogik in der beruflichen Integrationsförderung (Band 2). Münster: Waxmann.

Egger, M., Dreher, T. \& Partner (2007). Vertiefungsstudie Bildungsangebote im Übergang von der obligatorischen Schule in die Berufsbildung. Bern: BBT. Zugriff am 27.02.2009 unter http://www.bbt.admin.ch/dokumentation/00335/00400/index.html?lang=de

Friese, M. \& Stöppler, R. (2009). Partizipation statt Prekarisierung. Kooperative Ansätze der Berufs- und Sonderpädagogik. Berufsbildung. Zeitschrift für Praxis und Theorie in Betrieb und Schule, 63 (115), 3-7.

Greinert, W.-D. (1998). Das «deutsche System» der Berufsausbildung. Baden-Baden: Nomos.

Grimm, K. \& Vock, R. (2007). Sozialpädagogik in der beruflichen Integrationsforderung (Band 1). Münster: Waxmann.

Hendrich, W. (2005). Erwerbsbiographische Gestaltungskompetenz als Perspektive für Berufspädagogik und Erwachsenenbildung. In B. Niemeyer (Hrsg.), Neue Lernkulturen in Europa? (S. 19-40). Wiesbaden: VS-Verlag. 
Hupka, S. \& Stalder, B. E. (2004). Die Situation junger Migrantinnen und Migranten beim Übergang SekI/SekII. In Schweizerische Konferenz der Gleichstellungsbeauftragten (Hrsg.), Achtung Gender. Ausbildungsverhalten von Mädchen und jungen Frauen (S. 79-94). Bern.

Kleve, H. (2006). Case Management. Eine methodische Perspektive zwischen Lebensweltorientierung und Ökonomisierung. In H. Kleve, B. Haye, A. Hampe-Grosser \& M. Müller (Hrsg.), Systemisches Case-Management (S. 40-56). Heidelberg: Carl Auer.

Kratzer, N. (2003). Arbeitskraft in Entgrenzung. Grenzenlose Anforderungen erweiterte Spielräume, begrenzte Ressourcen. Berlin: edition sigma.

Kraus, K. (2006). Vom Beruf zur Employability? Zur Theorie einer Pädagogik des Erwerbs. Wiesbaden: VS-Verlag.

Kraus, K. (2008). Beschäftigungsfähigkeit oder Maximierung von Beschäftigungsoptionen? Ein Beitrag zur Diskussion um neue Leitlinien für Arbeitsmarkt- und Beschäftigungspolitik. Friedrich-Ebert-Stiftung (Hrsg.). Bonn. Zugriff am 21.07.09 unter http://library.fes.de/pdf-files/wiso/05865.pdf

Kraus, K. (2010). Case Management Berufsbildung und Educational Governance in der Schweiz - Eine Fallstudie. Zeitschrift für Berufs- und Wirtschaftspädagogik, 106.

Kutscha, G. (2008). Übergangsforschung im Übergang. In D. Münk, K. Breuer \& T. Deißinger (Hrsg.), Berufs-und Wirtschaftspädagogik - Probleme und Perspektiven aus nationaler und internationaler Sicht (S. 71-81). Opladen: Barbara Budrich.

Lex, T., Gaupp, N., Reißig, B. \& Adamczyk, H. (Hrsg.). (2006). Übergangsmanagement: Jugendliche von der Schule ins Arbeitsleben lotsen. München: DJI Verlag.

Link, J. (1986). Noch einmal: Diskurs. Interdiskurs. Macht. Kulturrevolution. Zeitschrift für angewandte Diskurstheorie, 11, 4-7.

Link, J. \& Link-Heer, U. (1990). Diskurs/Interdiskurs und Literaturanalyse. Zeitschrift für Literaturwissenschaft und Linguistik, 20, 88-99.

Lisop, I. (2009). Neue pädagogische Professionalität durch Managementkompetenz, Transferund Kooperationskultur. In Sektion Berufs- und Wirtschaftspädagogik in der Deutschen Gesellschaft für Erziehungswissenschaft (Hrsg.), Memorandum zur Professionalisierung des pädagogischen Personals in der Integrationsförderung aus berufsbildungswissenschaftlicher Sicht, S. 36-44.

Meyer, T. (2003). Jugendliche mit Migrationshintergrund. In Bundesamt für Statistik (Hrsg.), Wege in die nachobligatorische Ausbildung (S. 111-120). Neuchâtel.

Moldaschl, M. \& Voss, G. G. (Hrsg.). (2003). Subjektivierung von Arbeit. München: Rainer Hampp.

Niemeyer, B. (2004). Benachteiligtenförderung zwischen Berufung und Profession. vBerufsund Wirtschaftspädagogik online bwp@, 6, 1-12. Zugriff am 21.07.09 unter http://www.bwpat.de/ausgabe6/niemeyer-a-bwpat6.shtml

Rauner, F. (2006). Reformprojekt Berufliche Bildung (Zweite Fassung). Vorlage für die 5. Sitzung der «Reformwerkstatt Berufliche Bildung NRW» am 06.06.06 in Düsseldorf. Universität Bremen, Institut Technik und Bildung.

Rützel, J. (2009). Integration durch Ausgrenzung? Das Nadelöhr duale Berufsausbildung. Berufsbildung. Zeitschrift für Praxis und Theorie in Betrieb und Schule, 63 (115), 2.

Schaffner, D. (2007). Junge Erwachsene zwischen Sozialhilfe und Arbeitsmarkt: biografische Bewältigung von diskontinuierlichen Bildungs- und Erwerbsverläufen. Bern: hep.

Schröer, W. (1999). Sozialpädagogik und die soziale Frage. Der Mensch im Zeitalter des Kapitalismus um 1900. Weinheim/München: Juventa.

Stratmann, K. W. (1992). Arbeiterjugend im Kaiserreich. In K. W. Stratmann (Hrsg.), Historische Berufsbildungsforschung. 9. Beiheft zur Zeitschrift für Berufs- und Wirtschaftspädagogik (S. 147-166). Stuttgart: Franz Steiner.

Wendt, W. R. (2007). Die Zukunft des Case Managements. Soziale Arbeit, 56, 460-468. 
Schlagworte: Berufsbildung, Case Management, Übergänge, Sozialpädagogik, Subjektivierung von Erwerbsarbeit, Flexibilisierung, Individualisierung

\title{
Case management en formation professionnelle - La transformation de la formation professionnelle face à la transformation du travail
}

\begin{abstract}
Résumé
De plus en plus de jeunes rencontrent des difficultés pour entrer dans la formation professionnelle et sur le marché du travail. Ceci touche fondamentalement la fonction d'intégration sociale de la formation professionnelle. Le «case management en formation professionnelle» (CMFP) devrait remédier à ces difficultés. L'analyse des concepts relatifs au CMFP montre que la justification de son implémentation s'appuie prioritairement sur des arguments se réferant à des problèmes sociaux, à desquestions de capacités d'accueil et de dysfonctionnements de la formation professionnelle. Les conclusions de l'analyse mettent en évidence que la subjectivation de la formation professionnelle engendrée par le CMFP est à saisir dans le contexte d'une transformation plus générale du travail salarié, car elle modifie fondamentalement les conditions d'intégration sociale. La compréhension élargie de la politique de transition s'avère en conséquence indispensable.
\end{abstract}

Mots clés: Formation professionnelle, case management, transition, pédagogie sociale, subjectivation du travail salarié, flexibilitation, individualisation

\section{Case Management nella formazione professionale: una riforma nel contesto della trasformazione del lavoro}

\section{Riassunto}

Un numero crescente di giovani incontra difficoltà nella transizione verso la formazione e all'entrata nel mondo del lavoro, il che contribuisce ad indebolire la funzione integrativa del sistema di formazione professionale. Il cosiddetto case management che dovrebbe porre rimedio al problema viene giustificato con riferimento a problemi di carattere politico-sociale e a disfunzionalità del sistema della formazione professionale. Inoltre per la comprensione del fenomeno va considerata l'individualizzazione del lavoro che modifica radicalmente le condizioni d'integrazione sociale. Occorre pertanto ampliare, secondo gli autori, i concetti relativi al problema della transizione.

Parole chiave: Formazione professionale, case management, transizione, pedagogia sociale, individualizzazione del lavoro, flessibilità 


\section{Case Management in Vocational Education - A Reform in the light of the transformation of work}

\section{Summary}

The number of young people who have difficulties making the transition from school to work is currently on the rise in Switzerland, a situation that also weakens the socially integrative function of vocational education. Reforms introducing case management, already known in the field of social work, into vocational education aim to help young people entering and completing vocational education, and also to strengthen its integrative function. This paper analyses the notion of case management in vocational education and shows that the justification for these reforms is related either to difficulties within the system of vocational education or to the broader problem of social cohesion. It also argues that the introduction of an individual-oriented approach such as case management into vocational education must be seen in the wider context of the transformation and individualisation of work.

Key words: School-to-work transition, vocational education and training (VET), case management, transformation of work, social work, individualisation 
